\title{
Peritoneal abscess resulting from a translocated copper intrauterine device
}

\author{
Jyoti Jatti, Sally James, Mark C James
}

\section{Introduction}

Translocation of an intrauterine device (IUD) is a widely reported and recognised complication of insertion. We describe an interesting location within a sterile anterior peritoneal abscess, presenting in an asymptomatic woman with an unplanned pregnancy, 1 year after its insertion. This case highlights the importance of checking that the IUD threads are present and the difficulties associated with establishing its location and the surgical retrieval of a translocated IUD.

\section{Case report}

A 21-year-old woman was fitted with a Nova-T ${ }^{\circledR} 380$ IUD 10 weeks after the delivery of her first child. There were no reported difficulties with its insertion at the family planning service. However, the woman reported to her general practitioner (GP) 4 days after its insertion, complaining of lower abdominal pain. On examination the threads were not visible and a perforation was suspected. An ultrasound scan reported some echogenic foci seen within the midline of the uterine cavity and suggested that these probably represented the presence of an IUD. No attempt was made to retrieve the threads and the woman was not referred on at that stage. Her left-sided lower abdominal pain persisted on and off over the following months but appeared to be settling. One year after the insertion of the IUD the woman was surprised to find herself pregnant. This was an unplanned and unwanted pregnancy and she subsequently underwent a surgical termination. At the time of the procedure, which was planned through the Pregnancy Advisory Service, there was no evidence of an IUD.

Following the termination an abdominal X-ray, arranged by her GP, demonstrated an IUD within the pelvic cavity to the left of the midline (Figure 1). At this point the woman was referred to the gynaecology department and admitted for a hysteroscopy and laparoscopy. The uterine cavity was normal with no evidence of an IUD or a perforation site and it was not possible to locate the device during the laparoscopy. Interestingly, at laparoscopy a $5 \mathrm{~cm}$ retroperitoneal cystic area was reported anteriorly in the abdominal wall. This was in the region of the left iliac fossa and the woman's abdominal discomfort was attributed to it. An ultrasound scan was arranged to ascertain the nature of this cystic area. The scan reported a cystic area lying immediately under the anterior abdominal wall to the left

J Fam Plann Reprod Health Care 2007; 33(1): 57-58

(Accepted 12 July 2006)

Department of Gynaecology, Gloucestershire Royal Hospital, Gloucester, UK

Jyoti Jatti, MD, DFFP, Registrar

Mark C James, MD, MRCOG, Consultant Obstetrician and

Gynaecologist

Department of Radiology, Gloucestershire Royal Hospital, Gloucester, UK

Sally James, FRCR, Consultant Radiologist

Correspondence to: Mr M C James, Department of Obstetrics and Gynaecology, Gloucestershire Royal Hospital, Gloucester GL1 3NN, UK. E-mail: mark.james@glos.nhs.uk

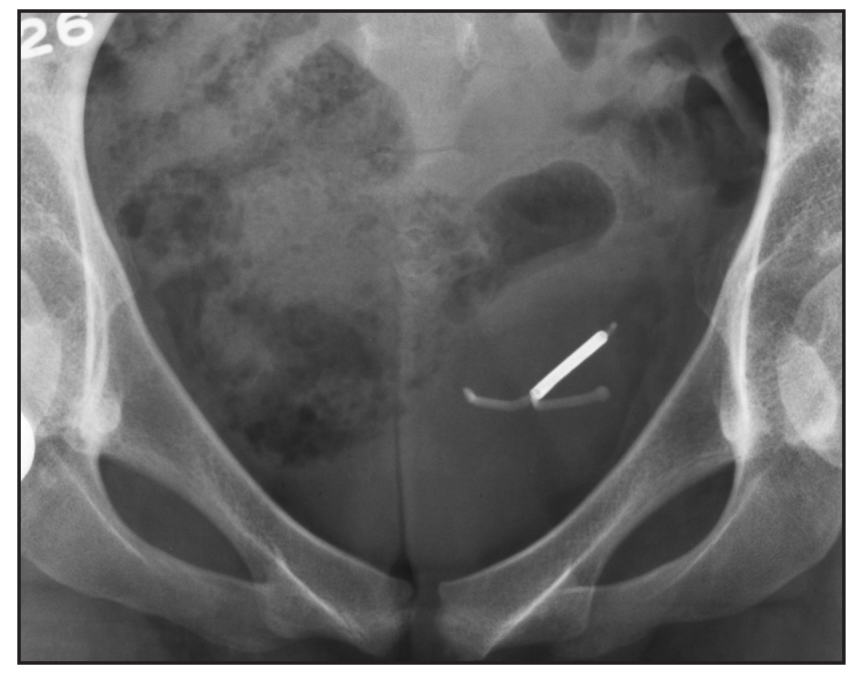

Figure 1 Radiograph of the abdomen showing the intrauterine device to the left of midline

of the midline in the left iliac fossa. It also reported the presence of an IUD within the cyst (Figure 2).

A repeat laparoscopy was performed, allowing incision of the cyst and retrieval of the missing IUD. The woman was discharged home the following morning and a followup appointment was made with the family planning service. A culture of the IUD showed no pathogenic organisms.

\section{Discussion}

The differential diagnosis of missing threads includes unnoticed expulsion, ascent of the tail into the cavity, and perforation or translocation of the IUD. Translocation of an IUD to an extrauterine site in the peritoneal cavity is uncommon. The various sites of a translocated IUD reported in the literature include the broad ligament, culde-sac, omentum, sigmoid colon, rectum, urinary bladder, uterovesical fold and space of Retzius. Serious consequences have been described such as damage to the viscera (i.e. bowel, kidney), uterine lesion and/or peritonitis.

The incidence of uterine perforation due to the use of modern IUDs is estimated to be 1 per 3000 insertions. ${ }^{1}$ The

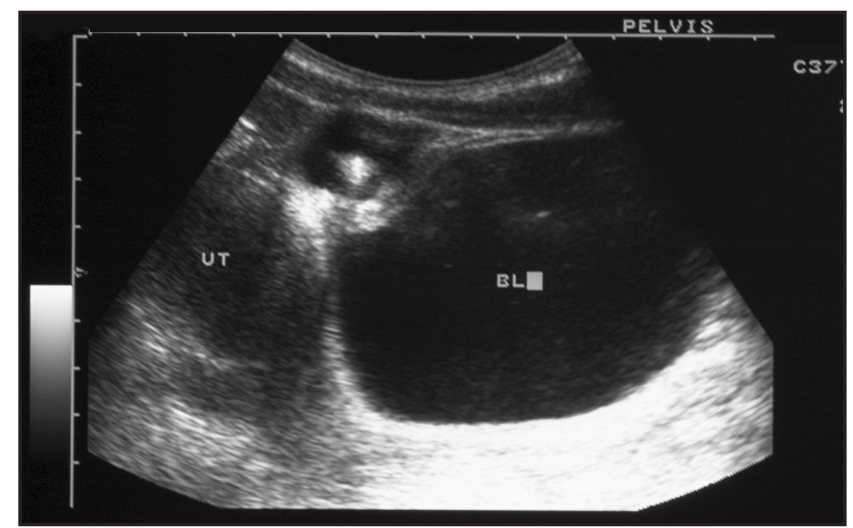

Figure 2 Pelvic ultrasound image demonstrating a cystic area underneath the anterior abdominal wall and an end-on view of the intrauterine device within. BL, bladder; UT, uterus 
observational study on 17469 Multiload $^{\circledR} \quad \mathrm{Cu} 375$ insertions gave an incidence of 1.6 per 1000 insertions. $^{2}$ To help women in decision making regarding copper IUDs, the National Institute for Health and Clinical Excellence (NICE) recommends that information imparted to women should include contraceptive efficacy, risks and possible side effects. This guideline quotes less than 1 in 1000 in terms of risk of uterine perforation at the time of IUD insertion. ${ }^{3}$

During the puerperium, when the uterus is small and the uterine wall is thin, the risk of perforation increases. ${ }^{4}$ Established practice in UK has been to delay insertion until 6-8 weeks postpartum. The World Health Organization Medical Eligibility Criteria for Contraceptive Use (WHOMEC) recommends unrestricted use of an IUD four or more weeks postpartum in women who are breastfeeding, not breastfeeding or post-Caesarean section. ${ }^{5}$ WHOMEC suggests an increased risk of perforation if an IUD is inserted between 48 hours and 4 weeks postpartum and therefore the risks of insertion during this time generally outweigh the benefits. The care pathway in the use of IUDs by NICE recommends a routine follow up at 3-6 weeks or at first menses to check threads and exclude perforation. ${ }^{3}$

In the present case an early perforation was suspected and initial investigations indicated an IUD providing false reassurance. Lack of identifiable threads makes it impossible to ensure that the device is in situ and functioning as a contraceptive. If no threads are seen and uterine placement of the IUD cannot be confirmed clinically, an ultrasound scan should be arranged to locate the device and alternative contraception is recommended by the Faculty of Family Planning and Reproductive Health Care (FFPRHC). 6 However, if the scan cannot locate the IUD and the woman had not witnessed expulsion, a plain abdominal X-ray should be arranged to identify an extrauterine device. Hysteroscopy can be useful if the ultrasound scan is equivocal. ${ }^{6}$

Surgical retrieval of an extrauterine IUD is advised by the FFPRHC. ${ }^{6}$ The scientific literature strongly recommends removal of an extrauterine IUD even if its migration has not given rise to any clinical symptoms. IUDs in the abdomen, especially copper-containing ones, can result in the development of adhesions, and infection due to carrying bacteria from the insertion process. ${ }^{7}$

The technique for removing a translocated IUD will depend on its location, extent of adhesions and the experience of the operator. This might involve hysteroscopy, colpotomy, laparoscopy and laparotomy or cystoscopy for retrieval of intravesical IUDs. The feasibility of IUD retrieval via the laparoscope depends on the extent of the attachment of the device to intraperitoneal structures, particularly vascular and intestinal, and also the ability of the laparoscopist to spot the device within the peritoneal cavity. ${ }^{8}$ Laparoscopic removal is difficult when the IUD is in the omentum and cannot be seen. In the present case retrieval was relatively straightforward once the site had been established.

\section{Statements on funding and competing interests Funding None identified. \\ Competing interests None identified.}

References

1 Singh M. IUDs and transmigration - putting an old concern to rest. IPPF Med Bull 1995; 29: 2-3.

2 Harrison-Woolrych M, Ashton J, Coulter D. Uterine perforation on intrauterine device insertion: is the incidence higher than previously reported? Contraception 2003; 67: 53-56.

3 National Institute for Health and Clinical Excellence (NICE). Long-acting Reversible Contraception (Clinical Guideline 30). London, UK: NICE, October 2005.

4 Kiilholma P, Makinen J, Maenpaa J. Perforation of the uterus following IUD insertion in the puerperium. Adv Contracept 1990; 6: 57-61.

5 World Health Organization (WHO). Medical Eligibility Criteria for Contraceptive Use. Geneva, Switzerland: WHO, 2000.

6 Faculty of Family Planning and Reproductive Health Care Clinical Effectiveness Unit. FFPRHC Guidance (January 2004). The copper intrauterine device as long-term contraception. J Fam Plann Reprod Health Care 2004; 30: 29-42.

7 Soderstrom RM. Trailing and treating the wandering IUD. Am J Gynecol Health 1989; 3(3-S): 33-34.

8 McKenna PJ, Mylotte MJ. Laparoscopic removal of translocated contraceptive devices. Br J Obstet Gynaecol 1982; 89: 163-165.

\section{BOOK REVIEW}

Fast Facts: Contraception (2nd edn). A Glasier, B Winikoff. Oxford, UK: Health Press Ltd, 2005. ISBN: 1-903734-46-0. Price: £15.00. Pages: 104 (paperback)

Most who read this Journal are familiar with the range of family planning methods available and are up to date enough not to need a small handbook such as this one. They will, however, often need to make available information to colleagues in primary care whose special interests lie elsewhere. Practice nurses and GP registrars will need to find succinct advice.

This book is part of the Fast Facts Series aimed at the UK and North American market. It is small, easy on the eye, and pleasant to hold. One of its authors is a director of family planning in Scotland and the other works in New York. Neither works in UK general practice. This is apparent.

The book covers all the contraception topics that you would expect, but with the complication of discussing many North American products. In some ways this is interesting.For the people here in the UK most likely to need such a brief guide, this just makes the book difficult to use. Key points are in coloured boxes, which are useful, but references are highlighted. Illustrations are easy on the eye. A large diagram of a GyneFix ${ }^{\circledR}$, and a photograph of a Mirena ${ }^{\circledR}$, without a picture of a standard copper intrauterine device (IUD), reflects the fact that this book has not been directed at UK primary care. It is part of a publisher's series

There are some surprising omissions. Counselling before referral for sterilisation, before IUD insertion and after childbirth is not clearly covered. Controlling infection transmission is a part of any sexual health consultation, as are psychosexual issues that present. Relatively little is said about these. Contraception cannot be treated as a topic in isolation.

The book tells us that "women whose lifestyle puts them at risk of sexually transmitted infections should be screened before IUD insertion". In UK general practice, assumptions are not made about patients and it would be more usual to screen everyone.

The style is very dry and dull, unnecessarily so for such a sexy subject. Contrast this book with John Guillebaud's Contraception Today, which is eloquently written. For the hurried enquirer, some of the information is cumbersome to access.

I do not think that this book is the first choice for practitioners in the UK.

Reviewed by Helen Grace Gibson, MRCGP, DFFP General Practitioner, Kingston-upon-Hull, UK

\section{Visit the Faculty Website at www.ffprhc.org.uk}

